\title{
Skin lesions in returning travellers
}

\author{
Krzysztof Korzeniewski ${ }^{1}$, Dariusz Juszczak ${ }^{2}$, Janusz Jerzemowski ${ }^{3}$ \\ ${ }^{1}$ Department of Epidemiology and Tropical Medicine, Military Institute of Medicine, Warsaw, Poland \\ ${ }^{2}$ Commander of the $7^{\text {th }}$ Navy Hospital, Gdansk, Poland \\ ${ }^{3}$ Health Science Institute, Pomeranian University, Slupsk, Poland
}

\begin{abstract}
Skin lesions, apart from diarrhoeas, fever of unknown origin, and respiratory tract infections belong to the most frequent medical problems in travellers returned from tropical and subtropical destinations, accounting more than $10 \%$ of reported cases. Most dermatoses have their clinical onset during travel, although some of them can occur after return. Travel-related dermatological problems can have a wide spectrum of clinical picture, from macular, popular or nodular rash, linear and migratory lesions, to plaques, vesicles, bullae, erosions or ulcers. Skin conditions in returning travellers may be of infectious and non-infectious aetiologies. Infectious lesions may be originally tropical (e.g. dengue, chikungunya, schistosomiasis, leishmaniasis, myiasis, tungiasis, loiasis), although the majority are cosmopolitan (arthropod bites, sunburns, allergic rashes). The evaluation of skin lesions depends on many factors, including immune status of patients, use of medicines, exposure on health hazards (fauna, flora, risky behaviours), as well as the time, duration and location of travel. As the number of travellers to tropical and subtropical destinations has been continuously rising, the number of skin illnesses has also been increasing. This means that specialists in travel medicine need to extend their knowledge of epidemiology, clinical features and diagnosis of travel-related health problems including skin lesions in returning travellers.
\end{abstract}

(Int Marit Health 2015; 66, 3: 173-180)

\section{Key words: skin lesions, returning travellers, tropics}

\section{INTRODUCTION}

Skin lesions, apart from diarrhoeas, fever of unknown origin, and respiratory tract infections belong to the most frequent medical problems in travellers returned from tropical and subtropical destinations [1]. According to the data obtained from travel medicine clinics [2-4], skin illnesses are the third most common reason for consultation and treatment among returning travellers, accounting for more than $10 \%$ of cases $[3,5]$. Potentially, $10 \%$ of travel-related skin conditions may be serious enough to lead to hospitalisation [6]. Most dermatoses have their clinical onset during travel, with the remainder tending to occur a median of 1 week after return [7]. Travel-related dermatological problems can have a wide spectrum of clinical symptoms. The most common are macular, popular, nodular, linear, erosion and ulcerative lesions. Macular lesions may oc- cur due to drug reactions and superficial mycoses (tinea versicolor, tinea corporis). Papular lesions can appear as a result of insect bites or hypersensitivity reactions. Nodular lesions are connected with bacterial infections (pyodermas) or can rise in the process of parasitic infection, e.g. myiasis, tungiasis or filariasis. Linear lesions may appear in parasitic infections (cutaneous larva migrans, larva currens) or noninfectious conditions (e.g. phytophotodermatitis). Erosive and ulcerative lesions originally occur as pyodermas (caused by Staphylococcus aureus, Streptococcus pyogenes) or parasitic infection (cutaneous leishmaniasis), and secondarily as a result of scratching itchy lesions (e.g. arthropod bites) or superficial injuries of the skin. Dermatological manifestations in returned travellers fall into two categories: lesions associated with fever (rash in the process of viral infections, e.g. dengue, chikungunya, secondary 
bacterial infections, rickettsial infections) and those not associated with fever (most dermatoses). The diagnosis of skin illnesses is based on the type of lesions (macular, popular, nodular, linear, ulcerative), location (exposed vs. unexposed), patient's history (contact with insects, animals, humans, cosmetics, drugs, etc.), and associated symptoms (fever, pain, pruritus). It is important to determine if the skin problems are travel-related [1]. Skin conditions in returning travellers can be both of infectious and non-infectious aetiologies. Infectious skin lesions may be tropical in origin (25\%), although the majority are cosmopolitan [4]. Some of them can be the result of insects' bites, sunburn, bacterial infections or allergic rash. Travel rashes may be acquired from exposure to sun, heat, medicines, insects, marine life and/or a wide range of infectious agents: viruses, bacteria, fungi and parasites [8]. The predominant skin symptom is an important clue - itching suggests an allergic aetiology, tenderness at the infection site suggests infection [9]. The evaluation of skin lesions in travellers depends on many factors, including immune status of patients, use of medicines (taken during travel), time and duration of travel, destination and exposure to health hazards (fauna and flora, risky behaviors, e.g. extreme sports). The risk for dermatological infections increases among patients with AIDS, organ transplant recipients, patients undergoing chemotherapy or taking immunomodulatory medications, elderly people, diabetics, pregnant women [10].

\section{EPIDEMIOLOGY}

International tourist arrivals have increased from 25 million in 1950 to 278 million in 1980, 527 million in 1995, and 1133 million in 2014. According to the United Nations World Tourism Organisation (UNWTO), the number of international tourist arrivals is expected to increase by $3.3 \%$ a year and to reach 1.8 billion by 2030 [11]. Along with the increasing number of travellers, a significant growth in travel-related illnesses is observed. Dermatoses are a leading cause of health problems in travellers including infectious illnesses of exotic or cosmopolitan origin as well as environmental skin manifestations. Research results illustrate that the leading dermatological problems observed during travel are infections and environmental skin illnesses (arthropod-induced pruritic dermatitis, sunburns, marine-associated dermatitis, and superficial injuries) [12].

According to the data obtained from the GeoSentinel Surveillance Network clinics, 4,594 of all returned travellers (mainly from Southeast Asia, sub-Saharan Africa and South America) consulted by specialists in tropical and travel medicine in the period from 1997 to 2006 were diagnosed with skin illnesses (18\% of all patients who sought medical care). The most common travel-related dermatoses were arthropod bites including secondary infections, pyoder- mas, allergic rashes, and cutaneous larva migrans. Arthropod-related skin illnesses (insects bites, dengue, cutaneous leishmaniasis) accounted for 31\% of all dermatological diagnoses; $15 \%$ dermatoses were of unknown aetiology (rash, urticarial, pruritus) [8]. The GeoSentinel Network data from over 42 thousand patients worldwide (from 53 travel/tropical clinics located in 24 countries), who were evaluated after foreign travel, revealed that a skin disorder diagnosis was made in $19.5 \%$ of patients presenting to physicians, which was the third most common diagnosis after gastrointestinal (34\%) and febrile illnesses (23\%) [13]. Studies from Maldives and Fiji $[14,15]$ revealed that dermatoses were the most frequent illnesses in tourists, with sunburns, superficial injuries (including those due to contact with marine creatures), and skin infections reported most often. In the study of Freedman et al. [2], involving more than 17 thousand returning travellers, skin diseases were the third most common cause of health problems after febrile illnesses and acute diarrhoeas. The most common causes of dermatological problems were arthropod bites (with or without secondary infection), cutaneous larva migrans, allergic reactions, and pyodermas. Herbinger et al. [3] studied the data of over 34 thousand travellers returning from tropical and non-tropical countries to Germany in the period 1999-2009. The tourists' main destinations were Asia (40\%), Africa (27\%), and Latin America (21\%). According to the research results $12.2 \%$ of all health problems were skin illnesses. The leading causes of skin manifestations were arthropodal (23\%), bacterial (22\%), helminthic (11\%), protozoan (6\%), viral (6\%), allergic (5\%), and fungal (4\%). The most frequently diagnosed skin illnesses were insect bites (17\%), cutaneous larva migrans (8\%), cutaneous leishmaniasis (2.4\%), dengue fever (1.5\%), rickettsioses (1.3\%), myiasis $(0.8 \%)$, filarioses $(0.7 \%)$, schistosomiasis $(0.6 \%)$, and tungiasis (0.6\%). In the study of Solomon et al. [16], realised during 10 years (1998-2008) in Israel, 2,834 post-travel patients were examined, of whom $19 \%$ suffered from skin disorders. Among them 53\% suffered from infectious (tropical: cutaneous larva migrans, cutaneous leishmaniasis, myiasis; non-tropical: pyodermas, cellulitis) and $47 \%$ from noninfectious lesions. The most common group in this study were tropical diseases (63.5\%), and among them leishmaniasis was a leading illness.

Skin problems develop as a result of a variety of factors: exposure to new medications, extremes of temperature and humidity, exposure to plant or animal toxins. Skin conditions may be associated with the length of stay and environmental risks. Travellers who stay in urban centres in high standard accommodation for a brief period are much less likely to acquire a local skin disorders than those living with locals in rural areas for a long time [8]. Owing to the complexity of the diagnostic and therapeutic process, it is important to be 
Table 1. Skin lesions associated with travel-related infections. Source: $[4,19]$

\begin{tabular}{ll}
\hline Skin lesions & Infection \\
\hline Maculopapular rash & Dengue, chikungunya, schistosomiasis, Rickettsia spp., superficial fungal infections \\
Papular rash & Scabies, blastomycosis, histoplasmosis \\
Nodular rash & Myiasis, tungiasis \\
Vesicular/bullous rash & Herpes simplex, Herpes zoster \\
Linear lesions & Cutaneous larva migrans, strongyloidiasis (larva currens) \\
Migratory skin lesions & Cutaneous larva migrans, strongyloidiasis (larva currens), loiasis \\
Erosions/ulcers & Leishmaniasis, pyodermas \\
Lymphangitis & Sporotrichosis, leishmaniasis \\
Jaundice & Malaria, leptospirosis \\
Haemorrhagic lesions & Dengue, leptospirosis, meningococcal infection, Rickettsia spp.
\end{tabular}

Table 2. Causes of localized dermatoses in travellers according to the primary skin lesions. Source: [20]

\begin{tabular}{ll}
\hline Skin lesions & Causes of dermatoses \\
\hline Papules and nodules & Noninfectious causes (arthropod bites) \\
& Bacterial infection (pyodermas, mycobacterial infection) \\
& Parasitic infection (leishmaniasis, tungiasis, myiasis, schistosomiasis) \\
& Vungal infection (mycetoma, sporotrichosis) \\
Erythematous plaque & Bacterial infection (pyodermas, cellulitis) \\
& Parasitic infection (schistosomiasis, leishmaniasis) \\
& Fungal infection (dermatophytosis) \\
Vesicles and bullae & Noninfectious causes (sunburn, contact dermatitis, irritant dermatitis, arthropod bites) \\
& Bacterial infection (impetigo) \\
& Parasitic infection (cutaneous larva migrans) \\
& Viral infection (Herpes simplex, Herpes zoster) \\
& Noninfectious causes (spider bite) \\
Ulcers & Bacterial infection (pyodermas, syphilis, tularaemia, anthrax) \\
& Parasitic infection (leishmaniasis, sporotrichosis) \\
& Fungal infection (mycetoma, chromomycosis) \\
& Viral infection (Herpes zoster)
\end{tabular}

aware of the epidemiology and clinical features of dermatoses occurring among patients returning from various travel destinations, especially from the tropics and subtropics.

\section{CLINICAL SYMPTOMS OF TRAVEL-RELATED DERMATOSES}

Returned travellers often present to medical facilities with unique clinical manifestations. Dermatological manifestations of travel-related health problems are particularly problematic because of a broad differential diagnosis and physicians' unfamiliarity with rare diseases [17]. The spectrum of travel-related dermatoses seems to be closely related to the geographic area visited and the onset of signs and symptoms is related to the date of return. Sunburns, arthropod-related reactions and superficial injuries most often occur during the patient's stay abroad and are most common in hot-climate seaside destinations. Skin infections, particularly pyodermas, are cosmopolitan and are a common cause of dermatoses during travellers' stay abroad as well as after their return [18]. Skin manifestations in travellers can be divided into two main categories: noninfectious (including arthropod bites, allergic reactions, sunburns, endogenous dermatological conditions) and infectious. Dermatoses can be further divided into infectious tropical (e.g. cutaneous, larva migrans, cutaneous leishmaniasis, schistosomiasis, myiasis, tungiasis, rickettsial infections, dengue, chikungunya, loiasis) and infectious non-tropical or cosmopolitan (pyodermas, scabies, superficial fungal infections, Herpes simplex, Herpes zoster) (Table 1) $[4,19]$. Causes of localised dermatoses according to the 
primary skin lesions are presented in Table 2 [20]. Some skin diseases, infectious and noninfectious, seen typically in a non-travel context, may be exacerbated by travel to the tropics. Examples include eczema, purulent and fungal infections which thrive in hot, humid environments [21].

Clinical symptoms of the most common dermatoses observed in travellers visiting tropical and subtropical destinations are discussed below.

Arthropod bites. Bites or stings from arthropods result in local skin lesions due to allergic reaction or toxin release. Arthropods include a wide group of fauna: insects, arachnids, crustaceans, mites, ticks, centipedes, and others [4]. Arthropod bites are extremely common and account for $10 \%$ of dermatoses in travellers returned from the tropics and $15-18.7 \%$ of dermatoses in travellers worldwide [8, 22]. Allergic reactions may occur in minutes to hours after a bite of a sting. A secondary bacterial infection after bite, e.g. because of scratching, may appear days later. Common presentations include pruritic papules (after mosquito or sand fly bite), eschar of ulcer (ticks, spider) [4].

Bacterial skin infections. The clinical spectrum ranges from impetigo (Staphylococcus aureus and Streptococcus spp. are the main reported etiological factors), folliculitis, ecthyma, erysipelas, abscess, to necrotising cellulitis [12]. A secondary infection after insect bites is also a common cause of bacterial skin infection [2]. According to Lederman et al. [8] bacterial skin infections (including pyodermas and infected insect bites) are the most common cause of dermatoses reported in the GeoSentinel Surveillance Network medical centres consulting thousands of travellers returning from the tropics, especially from African (Madagascar, Kenya, South Africa) and Asian continent (Philippines, Thailand, Sri Lanka).

Dengue/chikungunya. After malaria, dengue is the second most common cause of systemic febrile illness in returning travellers worldwide, especially in Central America and sub-Saharan Africa [2]. Clinically, in 30-50\% of dengue cases, except for fever, myalgia, headache, retro-orbital pain, maculopapular rash is seen. Diffuse erythema, petechiae, or haemorrhagic lesions can also be observed [23]. Most patients present with classic dengue fever and have benign febrile illness, but dengue haemorrhagic fever and dengue shock syndrome can also occur among returned travellers [20]. Similarly to dengue, in chikungunya (diagnosed from Africa and Asia, and recently in southern Europe and the Americas) in 50\% of the cases maculopapular rash is observed, located mainly in trunk or extremities [24]. Arthralgia, arthritis, or tenosynovitis can be also observed and may last several months to years after the onset of infection [25]. Chikungunya and dengue skin manifestations are difficult to differentiate. Rash is a common feature shared by both diseases, it looks similar and affects roughly the same number of patients in both cases. However, the diseases may be distinguished by other clinical variables, e.g. lymphopoenia is significantly more frequent in chikungunya, whereas leukopenia, neutropenia, and thrombocytopenia are significantly more common in dengue [20, 26]; shock and gastrointestinal haemorrhages are complications associated with dengue fever, whereas long-lasting arthralgia is a complication specifically associated with chikungunya $[27,28]$.

Schistosomiasis. The majority of travel-associated infections occur in travellers visiting sub-Saharan Africa (an estimated $85 \%$ of the world's cases of schistosomiasis are in African continent). Schistosoma haematobium and S. mansoni are distributed throughout Africa; only S. haematobium is found in the Middle East; S. japonicum is found in Indonesia, the Philippines and parts of China. Two other species, rarely reported in humans, are S. mekongi found in Cambodia and Laos, and S. intercalatum found in parts of Central and Western Africa [29]. Schistosoma may infect the urinary tract (S. haematobium) or the intestines (other species). Invasion of the skin by the cercariae present in the fresh water gives rise to a pruritic papular rash that appears a few hours after exposure and usually lasts less than $48 \mathrm{~h}$ (cercarial dermatitis, swimmer's itch). Another phase of the infection is an acute schistosomiasis (Katayama syndrome), with the most common symptoms such as fever (in $70 \%$ of cases), skin lesions, cough, malaise, headache, hepatosplenomegaly and marked eosinophilia. Fever might be the first symptom of the disease and can be accompanied by urticarial rash and angioedema. The urticarial rash appears in $30-50 \%$ of patients and may last for about 8 days. It is a diffuse migratory rash, which often prompts patients to seek immediate medical care, at which time the patient may be diagnosed as having "allergic reactions" and treated with antihistamines or glucocorticoids, without further evaluation [30].

Rickettsial infections. Rickettsioses are zoonotic bacterial infections transmitted to humans by arthropods. Regardless of the causative agent, most patients usually present with a benign febrile illness accompanied by headache, myalgia and skin lesions (rash, cutaneous eschar) [20]. Severe complications such as multiple organ failure and fatalities are occasionally seen [31]. The leading cause of rickettsiosis reported in travellers is African tick-bite fever (ATBF), transmitted by cattle Amblyomma ticks and caused by Rickettsia africae. ATBF is endemic in rural sub-Saharan Africa and frequently occurs in clusters among travellers during hunting, safaris, adventure races [32]. Rash can occur in $>40 \%$ of patients diagnosed with this infection [33]. The characteristic skin lesion is a black eschar, which is found at the site of inoculation of $R$. africae. Patients may also develop a more generalised maculopapular or vesicular 
rash [19]. Other rickettsial infections can be seen endemic worldwide (e.g. Orientia tsutsugamuschi in South and Southeastern Asia and the Western Pacific, Rickettsia conorii in Mediterranean Europe, Africa, and Asia, Rickettsia rickettsia in the Americas) and in their clinical picture, dermatological symptoms vary from focal lesions (eschar) to disseminated rashes, usually macular or petechial $[34,35]$.

Cutaneous leishmaniasis (CL). $90 \%$ of the world cases of cutaneous leishmanisis (1.5 million of new cases every year) are observed in Afghanistan, Algeria, Brazil, Peru, Saudi Arabia, Iran, Iraq and Syria [36]. Endemic foci of CL exist in southern France and Greece, periodic outbreaks have occurred in Spain [37-39]. CL is more common in rural areas, in settings ranging from rainforests to arid regions. However it is increasingly reported in urban and suburban areas, where a number of opportunistic hosts, such as dogs and donkeys, have become the reservoir of infection [36]. The types of skin lesions vary and may progress from papules, plaques, nodules to ulceration. The classic manifestation of cutaneous leishmaniasis is a predominantly painless ulcer with raised, indurated margins, along with granulation tissue or eschar [19]. Because the lesions are located at the site of the sand fly bite (vector of leishmaniasis), they are typically found on areas of exposed skin such as the extremities or face. The skin lesions can develop within weeks to months of exposure or even years later, often in the setting of trauma or immunosuppression. Left untreated, they may last months to years [40]. The average number of lesions varies from 1 to 3 and rarely exceeds 10 per patient. The cutaneous form of leishmaniasis is often benign and self-limiting [41]. In travellers returning from South America, where except for $\mathrm{CL}$, the mucocutaneous form of disease also occurs; the clinical spectrum of leishmaniasis is larger, ranging from ulcerative skin lesions to destructive mucosal inflammation [42].

Cutaneous larva migrans (CLM). Skin infection caused by hookworms, is one of the most common in travellers returning from the tropics [43], especially from sub-Saharan Africa, Asia, South America and the Caribbean (the most common countries include Brazil, Jamaica, Malaysia and Thailand) $[44,45]$. According to selective studies the rates of cutaneous larva migrans among travellers range from $5 \%$ up to $25 \%$ [2, $8,22,46]$. The incidence of CLM follows a seasonal pattern, with more infections occurring during the rainy seasons [47]. After direct contact with surface contaminated the faeces, e.g. soil, the hookworm larvae penetrate the skin and migrate in the superficial tissue (mainly in lower extremities as a result of walking barefoot on the beach or on sandy soil). The incubation period of CLM is usually a few days and rarely goes beyond 1 month [12]. The clinical picture manifests as an erythematous, linear or serpiginous track (approx. $3 \mathrm{~mm}$ wide and 15-20 mm in length; it may extend a few millimetres to a few centimetres daily), and can be associated with a pruritic popular (mostly) or vesiculobullous rash (up to $15 \%$ cases) $[48,49]$. The mean number of lesions per person varies from 1 to $3[49,50]$. Pruritus localised at the site of the eruption is reported in 98-100\% of patients [50]. Most cases present with symptoms within weeks of exposure, rare cases are present months later [51]. CLM is self-limited (hookworms die in the skin as they are unable to penetrate further), but migration can persist for weeks to months (usually 2-8 weeks) with associated pruritus and discomfort. When left untreated, the lesions of CLM can become infected with bacterial pathogens (mostly as a result of scratching the skin) leading to secondary pyodermas [52].

Strongyloidiasis (larva currens). Cosmopolitan skin infection caused by the nematode Strongyloides stercoralis. The prevalence of helminthiasis is the highest in tropical and subtropical regions. Humans become infected by contact with soil containing infectious larvae of S. stercoralis. The larvae penetrate the intact skin. Gastrointestinal (abdominal pain, diarrhoea), pulmonary (Löffler's syndrome), and skin symptoms appear as a result of parasite's migration through the host's body [53]. There are two types of dermatological manifestations in strongyloidiasis. One is a visible linear or serpiginous creeping lesion (larva currens) with associated pruritus, mostly around the anus and anywhere on the trunk [19]. Unlike cutaneous larva migrans, larva currens migrate much faster than hookworms at a rate of $2-10 \mathrm{~cm}$ per hour as opposed to centimetres per day. The cutaneous lesions tend to persist for only hours to days and then completely disappear, but the lesions may recur frequently [52]. The second form of skin lesions in strongyloidiasis is an urticarial rash in an individual who has already been sensitised. This urticarial rash occurs predominantly in the buttocks and around the waist, lasts 1-2 days and may recur as a first form frequently at regular intervals [53]. An important feature of S. stercoralis is an ability to complete its lifecycle within the human body, leading to continuing cycles of autoinfection [54]. It's important to notice that chronic or persistent infection may remain asymptomatic over decades and culminate in severe and potentially life-threatening S. stercoralis hyperinfection syndrome once an individual sustains immunosuppression (e.g. chemotherapy, organ transplantation) $[55,56]$.

Myiasis/tungiasis. Infestations related to the presence of larvae or maggots of flies in human tissue. Dermatoses occur mainly in tropical and subtropical regions (myiasis can also occur in temperate climate), particularly in sub-Saharan Africa, South and Central America, and the Caribbean. Myiasis most commonly presents as an erythematous furuncle at the site of invasion with botfly (Dermatobia hominis) and tumbu fly (Cordylobia anthropophaga) as the most 
common causative agents. The incubation period varies from 7 days to 3 weeks [22]. C. anthropophaga lesions are more commonly multiple, whereas $D$. hominis lesions usually number from 1 to 3 [57]. Clinically, pruritus and the moving sensation at the lesion site with lancinating pain are seen [58]. If not associated with a pre-existing wound, the typical lesion of myiasis is nodular. Also, the disease may present with furuncular lesions, and thus it can be mistaken for a staphylococcal furuncle. The lesion usually has a small opening to allow for respiration, and patients sometimes report a sensation of movement within the skin. Risk factors for acquiring myiasis include open wounds and peripheral vascular disease [59].

Tungiasis is a result of penetration of the skin (more superficial than in myiasis) by sand or jigger flea (Tunga penetrans), usually between the toes or under toenails. The infection is acquired by walking barefoot in regions where T. penetrans is found (usually beaches or sandy soil). Animal reservoirs include dogs, cats, pigs, and rats [60]. The tiny female flea (approx. $1 \mathrm{~mm}$ ), burrows into the outer layer of the skin, generating the characteristic white papule or nodule with a central black point [61]. The burrowing process is asymptomatic, but the gravid female increases in size to up to $1 \mathrm{~cm}$ in about 2 weeks, creating a pruritic or painful nodular lesion from which eggs and faeces are excreted [62]. The flea usually dies and is sloughed with the host's skin over several weeks [63].

Loiasis. Filarial parasitic infection present in Western and Central Africa transmitted by bloodsucking flies. Most infections are asymptomatic. The classical loiasis caused by filaria (adult worm, $3-7 \mathrm{~cm} \times 0.4 \mathrm{~mm}$ ), migrating under the conjunctiva of the eye is rarely observed, as the visible passage of the worm only lasts 10-15 min [56]. More often, symptomatic loaiasis manifest as localised angioedema in response to subcutaneous migration of parasites [64]. The most frequent symptoms are recurrent subcutaneous soft tissue swellings (Calabar swellings) and chronic pruritus. Calabar swellings are painless, non-pitting angioedemas, most commonly observed on the hands, wrists and forearms, lasting a few hours to several days [56].

\section{SUMMARY}

Skin lesions are the third most common health problems in returning travellers, accounting for more than $10 \%$ of reported cases. Dermatoses have a wide spectrum of clinical symptoms, from macular, popular, nodular, linear, to erosion and ulcerative lesions. Tropical illnesses make up about one quarter of dermatological infections or infestations (mainly cutaneous larva migrans, myiasis, tungiasis, cutaneous leishmaniasis) and the remainder are cosmopolitan (arthropod bites, bacterial skin infections, allergic reactions, animal bites, superficial fungal infections). Differential diagnoses of skin illnesses depend on the patient's travel history (place of stay and duration of journey, local endemicity, certain diseases) and the clinical picture of dermatoses. Some dermatological problems can be self-limited, because of that some travellers do not seek medical assistance during and after journey. It must be remembered that dermatoses tend to develop much faster and more frequently in hot climate zones which is associated with increased secretion of sweat and sebum. In tropical regions travellers are exposed to a much larger number of insects (vectors of arthropod-borne diseases) than in temperate climate areas, they are also exposed to contaminated soil and water which translates into increased prevalence of health problems presenting with dermatological symptoms. Because the number of travellers to tropical and subtropical destinations has been continuously rising, the number of skin illnesses has also been increasing. This means that specialists in travel medicine need to extend their knowledge of epidemiology, clinical features and diagnosis of travel-related health problems including skin lesions in returning travellers.

\section{REFERENCES}

1. Keystone JS. Skin and soft tissue infections in returned travelers. In: Brunette GW (Ed.). CDC Health Information for International Travel 2016. Oxford University Press, New York 2015, pp. 507-512.

2. Freedman DO, Weld LH, Kozarsky PE et al. Spectrum of disease and relation to place of exposure among ill returned travelers. N Engl J Med 2006; 354: 119-130.

3. Herbinger KH, Siess C, Nothdurft HD, von Sonnenburg F, Löscher T. Skin disorders among travellers returning from tropical and non -tropical countries consulting a travel medicine clinic. Trop Med Int Health 2011; 16: 1457-1464.

4. O'Brien MB. A practical approach to common skin problems in returning travellers. Travel Med Infect Dis 2009; 7: 125-146.

5. Caumes E, Legros F, Duhot D, Cohen JM, Arnould P, Mosnier A. Health problems in returning travelers consulting general practitioners. J Travel Med 2008; 15: 457-459.

6. Caumes E, Carriere J, Guermonprez G, Bricaire F, Danis M, Gentilini M. Dermatoses associated with travel to tropical countries: a prospective study of the diagnosis and management of 269 patients presenting to a tropical disease unit. Clin Infect Dis 1995; 20: 542-548.

7. Caumes E. Skin diseases. In: Keystone J, Kozarsky P, Freedman D, Nothdurft H, Connor B (Eds.). Travel Medicine. Mosby Spain 2004, pp. 491-502.

8. Lederman ER, Weld LH, Elyazar IR et al. Dermatologic conditions of the ill returned traveler: an analysis from the GeoSentinel Surveillance Network. Int J Infect Dis 2008; 12: 593-602.

9. Juckett G. Infections, dermatologic conditions in the returned pediatric traveler. Pediatric Annals 2011; 7: 362-367.

10. Baaten GG, Geskus RB, Kint JA, Roukens AH, Sonder GJ, van den Hoek A. Symptoms of infectious diseases in immunocompromised travelers: a prospective study with matched controls. J Travel Med 2011; 18: 318-326.

11. United Nations World Tourism Organization. UNWTO Tourism Highlights 2015. Available at: www.unwto.org/annualreports. Accessed: 20 Aug 2015. 
12. Hochedez $P$, Caumes E. Common skin infections in travelers. J Travel Med 2008; 15: 252-262.

13. Leder K, Torresi J, Libman MD et al. GeoSentinel surveillance of illness in returned travelers, 2007-2011. Ann Intern Med 2013; 158: 456-468.

14. Raju R, Smal N, Sorokin M. Incidence of minor and major disorders among visitors to Fiji. In: Proceedings of the Second Conference on International Travel Medicine. International Society of Travel Medicine, Atlanta, GA 1992: 62.

15. Plentz K. Nontropical and noninfectious diseases among travelers in a tropical area during five year period (1986-1990). In: Proceedings of the Second Conference on International Travel Medicine. International Society of Travel Medicine, Atlanta, GA 1992: 77.

16. Solomon M, Benenson S, Baum S, Schwartz E. Tropical Skin Infections Among Israeli Travelers. Am J Trop Med Hyg 2011; 85: 868-872.

17. Zimmerman RF, Belanger ES. Skin infections in returned travelers: Update. Curr Infect Dis Rep 2015; 17: 10.

18. Caumes E. Skin diseases. In: Keystone J, Kozarsky P, Freedman D, Nothdurft H, Connor B (Eds.). Travel Medicine. Elsevier Ltd. USA 2008, pp. 523-535.

19. Eldridge M, Cohen SH. Cutaneous manifestations of infection in returning travelers. Curr Infect Dis Rep 2014; 16: 426. .

20. Hochedez P, Canestri A, Guihot A, Brichler S, Bricaire F, Caumes E. Management of travelers with fever and exanthema notably dengue and chikungunya infections. Am J Trop Med Hyg 2008; 78: 710-713.

21. Joyce MP. Skin diseases of travelers. Prim Care 2002; 29: 971-981

22. Caumes E, Carriere J, Guermonprez G, Bricaire F, Danis M, Gentilini M. Dermatoses associated with travel to tropical countries: a prospective study of the diagnosis and management of 269 patients presenting to a tropical disease unit. Clin Infect Dis 1995; 20: $542-548$

23. Wilder-Smith A, Schwartz E. Dengue in travelers. N Engl J Med 2015; 353: 924-932

24. Pialoux G, Gauzere BA, Jaureguiberry S, Strobel M. Chikungunya, an epidemic arbovirosis. Lancet Infect Dis 2007; 7: 319-327.

25. De Andrade DC, Jean S, Clavelou P, Dallel R, Bouhassira D. Chronic pain associated with the Chikungunya Fever: long lasting burden of an acute illness. BMC Infect Dis 2010; 10: 31.

26. Nicoletti L, Ciccozzi M, Marchi A et al. Chikungunya and dengue viruses in travelers. Emerg Infect Dis 2008; 14: 177-178.

27. Simon F, Parola P, Grandadam M et al. Chikungunya infection: an emerging rheumatism among travelers returned from Indian Ocean islands. Report of 47 cases. Medicine (Baltimore) 2007; 86: 123-137.

28. Nimmannitya S, Halstead SB, Cohen SN, Margiotta MR. Dengue and chikungunya virus infection in man in Thailand, 1962-1964. I. Observations on hospitalized patients with hemorrhagic fever. Am J Trop Med Hyg 1969; 18: 954-971.

29. Brunette GW (Ed.). CDC Health Information for International Travel. Yellow Book 2016. In: Montgomery S. Schistosomiasis. Centers for Disease Control and Prevention. Oxford University Press, New York 2015, pp. 303-307.

30. Schwartz E. Schistosomiasis. In: Schwartz E (Ed.). Tropical diseases in travelers. Wiley-Blackwell, Oxford 2009, pp. 229-242.

31. McGill PE. Viral infections: alpha-viral arthropathy. Baillieres Clin Rheumatol 1995; 9: 145-150.

32. Fournier PE, Roux V, Caumes E, Donzel M, Raoult D. Outbreak of Rickettsia africae infections in participants of an adventure race in South Africa. Clin Infect Dis 1998; 27: 316-323.
33. Raoult D, Fournier PE, Fenollar F et al. Rickettsia africae, a tick-borne pathogen in travelers to sub-Saharan Africa. N Engl J Med 2001; 344: 1504-1510.

34. Jensenius M, Fournier PE, Raoult D. Tick-borne rickettsioses in international travellers. Int J Infect Dis 2004; 8: 139-146.

35. Jensenius M, Fournier PE, Raoult D. Rickettsioses and the international traveler. Clin Infect Dis 2004; 39: 1493-1499.

36. Mansueto P, Seidita A, Vitale G, Cascio A. Leishmaniasis in travelers: A literature review. Travel Med Infect Dis 2014; 12: 563-581.

37. Lachaud L, Dedet JP, Marty P et al. Surveillance of leishmaniases in France, 1999 to 2012. Euro Surveill 2013; 18: 20534.

38. Gkolfinopoulou K, Bitsolas N, Patrinos S et al. Epidemiology of human leishmaniasis in Greece, 1981-2011. Euro Surveill 2013; 18: 20532.

39. Arce A, Estirado A, Ordobas M et al. Re-emergence of leishmaniasis in Spain: community outbreak in Madrid, Spain, 2009-2012. Euro Surveill 2013; 18: 20546.

40. Shirian S, Oryan A, Hatam GR, Panahi S, Daneshbod Y. Comparison of conventional, molecular, and immunohistochemical methods in diagnosis of typical and atypical cutaneous leishmaniasis. Arch Pathol Lab Med 2014; 138: 235-240.

41. Blum J, Desjeux P, Schwartz E, Beck B, Hatz C. Treatment of cutaneous leishmaniasis among travellers. J Antimicrob Chemother 2004; 53: 158-166.

42. Schwartz E, Hatz C, Blum J. New world cutaneous leishmaniasis in travellers. Lancet Infect Dis 2006; 6: 342-349.

43. Brenner MA, Patel MB. Cutaneous larva migrans: the creeping eruptions. Cutis 2003; 72: 111-115.

44. Bowman DD, Montgomery SP, Zajac AM, Eberhard ML, Kazacos KR. Hookworms of dogs and cats as agents of cutaneous larva migrans. Trands Parasitol 2010; 26: 162-167.

45. Hochedez P, Caumes E. Hookwork-related cutaneous larva migrans. J Travel Med 2007; 14: 326-333.

46. Ansart S, Perez L, Jaureguiberry S, Davis M, Bricaire F, Caumes E. Spectrum of dermatoses in 165 travelers returning from the tropics with skin diseases. Am J Trop Med Hyg 2007; 76: 184-186.

47. Heukelbach J, Jackson A, Ariza L, Feldmeier H. Prevalence and risk factors of hookworm-related cutaneous larva migrans in a rural community in Brazil. Ann Trop Med Parasitol 2008; 102: 53-61.

48. Heukelbach J, Feldmeier H. Epidemiological and clinical characteristics of hookwarm-related cutaneous larva migrans. Lancet Infect Dis 2008; 8: 302-309.

49. Davies HD, Sakuls P, Keystone JS. Creeping eruption. A review of clinical presentation and management of 60 cases presenting to a tropical disease unit. Arch Dermatol 1993; 129: 588-591.

50. Bouchaud 0 , Houze S, Schiemann R et al. Cutaneous larva migrans in travelers: a prospective study, with assessment of therapy with ivermectin. Clin Infect Dis 2000; 31: 493-498.

51. Jelinek T, Maiwald H, Nothdurft HD, Loscher T. Cutaneous larva migrans in travelers: synopsis of histories, symptoms, and treatment of 98 patients. Clin Infect Dis 1994; 19: 1062-1066.

52. Zimmerman RF, Belanger ES. Skin infections in returned travelers: update. Curr Infect Dis Rep 2015; 17: 10.

53. Neumayr A, Hatz C, Blum J. Not to be missed! Differential diagnoses of common dermatological problems in returning travellers. Travel Med Infect Dis 2013; 11: 337-349.

54. Gill GV, Welch E, Bailey JW, Bell DR, Beeching NJ. Chronic strongyloides stercoralis infection in former British Far East prisoners of war. QJM 2004; 97: 789-795.

55. Mejia R, Nutman TB. Screening, prevention, and treatment for hyperinfection syndrome and disseminated infections caused by Strongyloides stercoralis. Curr Opin Infect Dis 2012; 25: 458-463. 
56. Simonsen PE. Filariasis. In: Cook GC, Zumla Al (Eds.). Manson's tropical diseases. Elsevier Ltd., London 2009, pp. 1477-1513.

57. Jelinek T, Nothdurft HD, Rieder N, Loscher T. Cutaneous myiasis: review of 13 cases in travelers returning from tropical countries. Int J Dermatol 1995; 34: 624-626.

58. Francesconi F, Lupi O. Myiasis. Clin Microbiol Rev 2012; 25: 79-105.

59. Fydryszewski NA. Myiasis: diagnosis, treatment and medical use of maggots. Clin Lab Sci 2013; 26: 76-81.

60. Karunamoorthi K. Tungiasis: a neglected epidermal parasitic skin disease of marginalized populations: a call for global science and policy. Parasitol Res 2013; 112: 3635-3643.
61. Haddad Jr V, Cardoso JL, Lupi O, Tyring SK. Tropical dermatology: venomous arthropods and human skin: part I. Insect J Am Acad Dermatol 2012; 67: e331-e314.

62. Lefebvre M, Capito C, Durant C, Hervier B, Grossi O. Tungiasis: a poorly documented tropical dermatosis. Med Mal Infect 2011; 41: 465-468.

63. Lefebvre M, Capito C, Durant C, Hervier B, Grossi O. Tungiasis: a poorly documented tropical dermatosis. Med Mal Infect 2011; 41: 465-468.

64. Padgett JJ, Jacobsen KH. Loiasis: Africa eye worm. Trans R Soc Trop Med Hyg 2008; 102: 983-989. 